\title{
DUALITY IN SUPERSYMMETRIC YANG-MILLS AND THE QUANTUM HALL EFFECT
}

\author{
BRIAN P. DOLAN \\ Department of Mathematical Physics, National University of Ireland, Maynooth, Ireland \\ and \\ School of Theoretical Physics, Dublin Institute for Advanced Studies, \\ 10, Burlington Road, Dublin, Ireland \\ bdolan@thphys.nuim.ie
}

Received 21 June 2006

\begin{abstract}
The evidence for the parallel roles played by the modular group in $\mathcal{N}=2$ supersymmetric Yang-Mills in $(3+1)$ dimensions and the quantum Hall effect in $(2+1)$ dimensions is reviewed. In both cases a subgroup of the full modular group acts as a map between different low energy phases of the theory, parametrised by a complex parameter in the upper-half-complex plane whose real part is a topological parameter and whose imaginary part is the coupling associated the kinetic term of the effective $U(1)$ gauge theory. In the case of the quantum Hall effect experimental evidence in favour of the modular action is also reviewed.
\end{abstract}

Keywords: Duality; supersymmetry; quantum Hall effect.

PACS Nos.: 11.10.Hi, 11.30.Pb, 12.60.Jv, 73.40.Hm

\section{Duality in Electromagnetism}

Maxwell's equations in the absence of sources

$$
\begin{array}{ll}
\nabla \times \mathbf{E}+\frac{\partial \mathbf{B}}{\partial t}=0, & \nabla \cdot \mathbf{E}=0, \\
\nabla \times \mathbf{B}-\frac{\partial \mathbf{E}}{\partial t}=0, & \nabla \cdot \mathbf{B}=0
\end{array}
$$

(using units in which $\varepsilon_{0}=\mu_{0}=c=1$ ) are not only symmetric under the Pioncaré group but also under the interchange of electric field $\mathbf{E}$ and the magnetic field $\mathbf{B}$, more specifically Maxwell's equations are symmetric under the map

$$
\mathrm{E} \rightarrow \mathbf{B} \quad \text { and } \quad \mathbf{B} \rightarrow-\mathbf{E} .
$$

This symmetry is known as duality, for any field configuration $(\mathbf{E}, \mathbf{B})$ there is a dual configuration $(\mathbf{B},-\mathbf{E})$. Duality is a useful symmetry, e.g. in rectangular waveguide problems, using this symmetry one can immediately construct a transverse magnetic mode once a transverse electric mode is known. 
When electric sources, i.e. a current $J^{\mu}$, are included this is no longer a symmetry but, in a seminal paper, Dirac ${ }^{1}$ showed that a vestige of (2) remains provided magnetic monopoles are introduced. By quantising a charged particle with electric charge $Q$, in a background magnetic field generated by a monopole with magnetic charge $M$, Dirac showed that single-valuedness of the wave-function requires that $Q M$ must satisfy the quantisation condition

$$
Q M=2 \pi \hbar n \Rightarrow M=\frac{2 \pi \hbar n}{Q},
$$

where $n$ is an integer. If $-e$ is the charge on an electron, then there is a fundamental unit of magnetic charge, namely $\frac{2 \pi \hbar}{e}$, which we shall denote by

$$
m=\frac{2 \pi \hbar}{e}
$$

and the allowed magnetic charges are integral multiples of $m$.

Since magnetic monopoles have never been observed, if they exist at all, they must be very heavy, much heavier than an electron, and so duality is not a manifest symmetry of the physics it is at best a map between two descriptions of the same theory. As $\alpha_{e}=\frac{e^{2}}{4 \pi \hbar} \approx \frac{1}{137}$ is so small, the "magnetic" fine structure constant

$$
\alpha_{m}=\frac{m^{2}}{4 \pi \hbar}=\frac{1}{4} \frac{1}{\alpha_{e}} \approx 34
$$

is very large and magnetic monopoles have very strong coupling to the electromagnetic field.

There is a generalisation of the Dirac quantisation condition for particles that carry both an electric and a magnetic charge at the same time, called dyons. If a dyon with electric charge $Q$ and magnetic charge $M$ orbits a second dyon with charges $Q^{\prime}$ and $M^{\prime}$, then Schwinger and Zwanziger showed that ${ }^{2}$

$$
Q M^{\prime}-Q^{\prime} M=2 \pi n \hbar .
$$

A dimensionless version of the Dirac-Schwinger-Zwanziger quantisation condition can be obtained by writing $Q=q e, Q^{\prime}=q^{\prime} e, M=p m$ and $M^{\prime}=p^{\prime} m$ giving

$$
q p^{\prime}-q^{\prime} p=n
$$

with $q, q^{\prime}, p, p^{\prime}$ and $n$ integers.

The simple $\mathbf{Z}_{2}$ duality map (5)

$$
2 \alpha_{e} \rightarrow 2 \alpha_{m}=\frac{1}{2 \alpha_{e}}
$$

can be extended to a much richer structure ${ }^{3,4}$ involving an infinite discrete group, the modular group $\mathrm{Sl}(2, \mathbf{Z}) / \mathbf{Z}_{2} \cong \Gamma(1)$, by including a topological term in the fourdimensional action,

$$
S=\int\left(-\frac{1}{4 e^{2}} F_{\mu \nu} F^{\mu \nu}+\frac{\theta}{32 \pi^{2}} \varepsilon^{\mu \nu \rho \sigma} F_{\mu \nu} F_{\rho \sigma}\right) d^{4} x .
$$


Defining the complex parameter

$$
\tau=\frac{\theta}{2 \pi}+\frac{2 i \pi}{e^{2}},
$$

using units with $\hbar=1,(8)$ generalises to the infinite set of maps

$$
\gamma: \tau \rightarrow \frac{a \tau+b}{c \tau+d},
$$

where $a, b, c$ and $d$ are any four integers satisfying the condition $a d-b c=10^{a}$ A general element of the modular group can be represented as a $2 \times 2$ matrix of integers,

$$
\gamma=\left(\begin{array}{ll}
a & b \\
c & d
\end{array}\right)
$$

with $\operatorname{det} \gamma=a d-b c=1$, so $\gamma \in \mathrm{Sl}(2, \mathbf{Z})$, and group multiplication then corresponds to matrix multiplication. Clearly $-\gamma$ has the same effect on $\tau$ as $\gamma$ does so the modular group is $\Gamma(1) \cong \mathrm{Sl}(2, \mathbf{Z}) / \mathbf{Z}_{2}$. The modular group is generated by two maps: one that we shall denote by $\mathcal{S}$

$$
\mathcal{S}: \tau \rightarrow-\frac{1}{\tau}
$$

reduces to (8) when $\theta=0$, and a second that we denote by $\mathcal{T}$

$$
\mathcal{T}: \tau \rightarrow \tau+1,
$$

is a consequence of the topological nature of the $\theta$ parameter, the partition function obtained from (9) is invariant under $\theta \rightarrow \theta+2 \pi$. A useful identity is $(\mathcal{S T})^{3}=1$.

Notice that the imaginary part of $\tau$ satisfies $\Im \tau>0$ (for stability $e^{2}>0$ ) and so $\tau$ parametrises the upper-half complex plane. Both $\mathcal{S}$ and $\mathcal{T}$ preserve the property that $\Im \tau>0$ and they generate the modular group acting on the upper-half complex plane.

\section{Duality in $\mathcal{N}=2, \mathrm{SU}(2)$ SUSY Yang-Mills}

Dirac considered a quantised particle moving in a fixed classical background field and modular transformations are unlikely to be useful in the full theory of QED when coupled electromagnetic and matter fields are quantised. Nevertheless Seiberg and Witten showed ${ }^{5}$ in 1994 that supersymmetry is a powerful constraint in fully quantised $\mathcal{N}=2$ supersymmetric Yang Mills theory: in the low-energy/longwavelength limit of the theory modular transformations of the complex coupling, or at least a subgroup of them, are relevant.

Consider SU(2) Yang Mills in four-dimensional Minkowski space with global $\mathcal{N}=2$ supersymmetry and, in the simplest case, no matter fields. ${ }^{\mathrm{b}}$ The field content

an Euclidean space a consequence of this is that the partition transform is related to a modular form and depends on topological invariants, the Euler characteristic and the Hirzebruch signature, in a well-defined manner. ${ }^{4}$

b There is by now a number of good reviews of $\mathcal{N}=2$ SUSY, see for example Refs. 6 and 7 . 
is then the $\mathrm{SU}(2)$ gauge potential, $A_{\mu}$; two Weyl spinors (gluinos) in a doublet of $\mathcal{N}=2$ supersymmetry, both transforming under the adjoint representation of $\mathrm{SU}(2)$, and a single complex scalar $\phi$, again in the adjoint representation. The bosonic part of the action is

$$
\begin{gathered}
S=\int d x^{4}\left\{-\frac{1}{4 g^{2}} \operatorname{tr}\left(F_{\mu \nu} F^{\mu \nu}\right)+\frac{\theta}{32 \pi^{2}} \varepsilon^{\mu \nu \rho \sigma} \operatorname{tr}\left(F_{\mu \nu} F_{\rho \sigma}\right)\right. \\
\left.+\frac{1}{g^{2}} \operatorname{tr}\left(\left(D_{\mu} \phi\right)^{\dagger} D^{\mu} \phi-\frac{1}{2}\left[\phi^{\dagger}, \phi\right]^{2}\right)\right\} .
\end{gathered}
$$

The fermionic terms (not exhibited explicitly here) are dictated by supersymmetry and involve Yukawa interactions with the scalar field but it is crucial that supersymmetry relates all the couplings and the only free parameters are the gauge coupling $g$ and the topological susceptibility $\theta$, all other couplings are in fact determined by $g$. Just as in electrodynamics these parameters can be combined into a single complex parameter

$$
\tau=\frac{\theta}{2 \pi}+i \frac{4 \pi}{g^{2}}
$$

and semiclassical arguments imply modular symmetry (11) on this parameter. ${ }^{5}$ Quantum effects reduce this to a smaller group.

Classically the Higgs potential is minimised by any constant $\phi$ in the Lie algebra of $\mathrm{SU}(2)$ such that $\left[\phi^{\dagger}, \phi\right]=0$, and since we can always rotate $\phi$ by a globally welldefined gauge transformation, we can always take $\phi=\frac{1}{2} a \sigma_{3}$ with $\sigma_{3}$ the usual Pauli matrix and $a$ a complex constant with dimensions of mass. The classical vacuum is thus highly degenerate and can be parametrised by $a$, a nonzero $a$ breaks $\mathrm{SU}(2)$ gauge symmetry down to $\mathrm{U}(1)$ (we are still free to rotate around the $\sigma_{3}$ direction) and $W^{ \pm}$bosons acquire a mass proportional to $a$, leaving one $\mathrm{U}(1)$ gauge boson (the photon) massless. At the same time the fermions and the scalar field also pick up masses proportional to $a$ except for the superpartners of the photon which are protected by supersymmetry and also remain massless. At the special point $a=0$ the gauge symmetry is restored to the full $\mathrm{SU}(2)$ symmetry of the original theory, Seiberg and Witten then argue that supersymmetry protects this degeneracy so that it is not lifted and is still there in the full quantum theory. At low energies, much less than the mass $a$ for a generic value of $a$, the only relevant degrees of freedom in the theory are the massless $\mathrm{U}(1)$ gauge boson and its superpartners (except for some special values of $a$ ).

There is a number of very important consequences of quantisation. Firstly, the point $a=0$ where full $\mathrm{SU}(2)$ symmetry is restored in the classical theory is inaccessible in the quantum theory. Secondly the effective low-energy coupling runs as a function of $a$, since $a$ is related to a mass, this is somewhat analogous to the Callan-Symanzik running of the QED coupling. The effective gauge coupling only runs for energies greater than $a$, for energies less than $a$ the running stops and at 
low energies it becomes frozen at its value $g^{2}(a)$. Using by now standard asymptotic freedom arguments in gauge theory, the low energy effective coupling $g^{2}(a)$ thus decreases at large $a$ and increases at small $a$. In fact the topological susceptibility $\theta$ also runs with $a$ (as a consequence of instanton effects ${ }^{5,8}$ ) and this can be incorporated into an $a$-dependence of $\tau, \tau(a)$. At large $a$ the imaginary part of $\tau$, $\Im \tau$, becomes large while at small $a$ it is small.

A better, gauge-invariant, parametrisation of the quantum vacua is given by $u=\operatorname{tr}\left\langle\phi^{2}\right\rangle$. For weak coupling (large $a$ ) $u \approx \frac{1}{2} a^{2}$, but $\left\langle\phi^{2}\right\rangle \neq\langle\phi\rangle\langle\phi\rangle$ for strong coupling (small $a$ ). By making a few plausible assumptions, including:

- the low energy effective action is analytic in $u$ except for isolated singularities (holomorphicity is closely linked with supersymmetry);

- the number of singularities is the minimum possible compatible with stability of the theory $(\Im \tau>0)$,

Seiberg and Witten argued ${ }^{5}$ that in the quantum theory the strong coupling regime $g^{2} \approx 0$ is associated not with $a=0$ but instead with two points in the complex $u$-plane, $u= \pm \Lambda^{2}$ where $\Lambda$ is, by definition, the QCD mass scale at which the gauge coupling diverges. Furthermore they found an explicit expression for the full low energy effective action and argued that new massless modes appear at the singular points $u= \pm \Lambda^{2}$, in addition to the photon and its superpartners. These new massless modes are in fact dyons, with the magnetic charge associated with non-perturbative aspects of the classical theory (solitons). Since $g \rightarrow \infty$ when $u= \pm \Lambda^{2}, \tau$ is real at these points. For example the point $\tau=0$ is associated with $u=\Lambda^{2}$ and the dyons have zero electric charge, they are in fact simple monopoles with monopole charge 1 . The point $\tau=1$ is associated with $u=-\Lambda^{2}$ and the dyons have unit electric charge, and monopole charge 1.

The full modular group $\Gamma(1)$ is not manifest in the quantum theory, rather Seiberg and Witten showed that the relevant map is (11) with both $b$ and $c$ constrained to be even. This is a subgroup of the full modular group, denoted $\Gamma(2)$ in the mathematical literature, and it is generated by

$$
\mathcal{T}^{2}: \tau \rightarrow \tau+2 \quad \text { and } \quad \mathcal{F}^{2}: \tau \rightarrow \frac{\tau}{1-2 \tau},
$$

where $\mathcal{F}^{2}=\mathcal{S}^{-1} \mathcal{T}^{2} \mathcal{S}$. ${ }^{\mathrm{c}} u$ itself is invariant under $\Gamma(2)$, but $\tau(u)$ is a multi-branched function of $u$.

Seiberg and Witten's $\Gamma(2)$ action commutes with the scaling flow as $u$ is varied. Taking the logarithmic derivative of $\tau(u)$ with respect to $u$, and imposing $a d-b c=1$, we see that

$$
u \frac{d \gamma(\tau)}{d u}=\frac{1}{(c \tau+d)^{2}} u \frac{d \tau}{d u} .
$$

${ }^{\mathrm{c}}$ We define $\mathcal{F}=\mathcal{S}^{-1} \mathcal{T} \mathcal{S}: \tau \rightarrow \frac{\tau}{1-\tau}$. 
Meromorphic functions $\tau(u)$ satisfying (18) are well studied in the mathematical literature and are called modular forms of weight -2 .

For Seiberg and Witten's expression for $\tau(u)$ it was shown in Refs. 9-12 that

$$
-u \frac{d \tau}{d u}=\frac{1}{2 \pi i}\left(\frac{1}{v_{3}^{4}(\tau)}+\frac{1}{v_{4}^{4}(\tau)}\right),
$$

where

$$
\vartheta_{3}(\tau)=\sum_{n=-\infty}^{\infty} e^{i \pi n^{2} \tau} \quad \text { and } \quad \vartheta_{4}(\tau)=\sum_{n=-\infty}^{\infty}(-1)^{n} e^{i \pi n^{2} \tau}
$$

are Jacobi $\vartheta$-functions. ${ }^{\mathrm{d}}$ Scaling functions for $\mathcal{N}=2$ SUSY Yang-Mills are also discussed in Ref. 14. At weak coupling, $g^{2} \rightarrow 0, \tau \rightarrow i \infty, \vartheta_{3}(\tau) \rightarrow 1$ and $\vartheta_{4}(\tau) \rightarrow 1$ so

$$
u \frac{d \tau}{d u} \approx \frac{a}{2} \frac{d \tau}{d a} \rightarrow \frac{i}{\pi}
$$

which is the correct behaviour of the $\mathcal{N}=2$ one-loop $\beta$-function for the gauge coupling,

$$
a \frac{d g}{d a} \approx-\frac{g^{3}}{4 \pi^{2}} .
$$

At weak coupling (large $a$ ), $a$ is proportional to the gauge boson mass and this flow can be interpreted as giving the Callan Symanzik $\beta$-function in the asymptotic regime. This interpretation is however not valid for finite $a$ for two reasons: firstly the statement that $a$ is proportional to the gauge boson mass is only valid at weak coupling and secondly because (19) diverges at strong coupling, $g \rightarrow \infty$ where $\tau \rightarrow 0 .{ }^{12}$ The latter difficulty can be remedied by defining a different scaling function which is still a modular form of weight -2 . Seiberg and Witten's expression for $\tau(u)$ can be inverted to give $u(\tau)^{10-12}$

$$
\frac{u}{\Lambda^{2}}=\frac{\vartheta_{3}^{4}+\vartheta_{4}^{4}}{\vartheta_{3}^{4}-\vartheta_{4}^{4}}
$$

which is invariant under $\Gamma(2)$ modular transformations. ${ }^{11,15}$ Equation (19) can therefore be multiplied by any ratio of polynomials in $u$ and we still have a modular form of weight $-2 .^{\circ}$ It is shown in Ref. 17 that the correct form of the Callan Symanzik $\beta$-function at $\tau=i \infty, \tau=0$ and $\tau=1$, up to a constant factor, is obtained by using the scaling function

$$
-\left(1-\frac{u}{\Lambda^{2}}\right)\left(1+\frac{u}{\Lambda^{2}}\right) u \frac{d \tau}{d u}=\frac{2}{\pi i} \frac{1}{\left(\vartheta_{3}^{4}(\tau)+\vartheta_{4}^{4}(\tau)\right)}
$$

and this is the only possible choice. The flow generated by (24) is shown in Fig. 1, there are fixed points on the real axis at $\tau=q / p$ where the massless dyons have

dDefinitions and relevant properties of the Jacobi functions can be found in the mathematics literature, for example Ref. 13.

'This point was stressed in Ref. 10. See, for example, Theorem 4.3.4 of Ref. 16. 


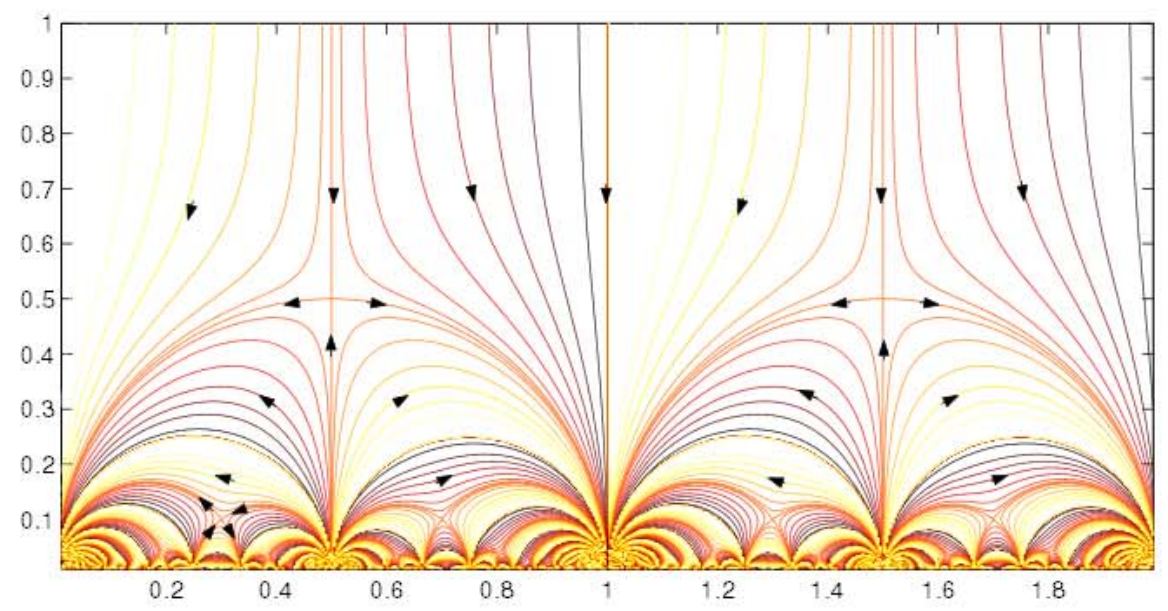

Fig. 1.

electric charge $q$ and magnetic charge $p$. Odd $p$ corresponds to attractive fixed points in the ir direction and even $p$ to attractive fixed points in the uv direction $(p=0$ corresponds to the original weakly coupled theory, $\tau=i \infty$ with massless gluinos). The repulsive singularities at $\tau=\frac{n+i}{2}$ with an odd integer $n$ occur for $u=0$ and are the quantum vestige of the classical situation where full $\mathrm{SU}(2)$ symmetry would be restored.

One point to note is that, since the scaling function (24) is symmetric under $u \rightarrow-u$, which is equivalent to $\tau \rightarrow \tau+1$, the full symmetry of the scaling flow is slightly larger than $\Gamma(2)$, it is generated by $\mathcal{F}^{2}$ and $\mathcal{T}$ and corresponds to matrices $\gamma$ such that $c$ in (11) is even. This group is often denoted by $\Gamma_{0}(2)$.

Observe also that there are semi-circular trajectories linking some of the ir attractive fixed points with odd monopole charge. These can all be obtained from the semi-circular arch linking $\tau=0$ and $\tau=1$ by the action of some $\gamma \in \Gamma_{0}(2)$, of the form (12) with $c$ even. Then $\tau_{1}=q_{1} / p_{1}=\gamma(0)=\frac{b}{d}$ and $\tau_{2}=q_{2} / p_{2}=\gamma(1)=\frac{a+b}{c+d}$ from which $b=q_{1}, d=p_{1}$ and $q_{2}=a+b, p_{2}=c+d$. Hence

$$
q_{2} p_{1}-q_{1} p_{2}=1,
$$

since $a d-b c=1$, giving a selection rule for transitions between ir attractive fixed points as $u$ is varied. This is clearly related to the Dirac Schwinger Zwanziger quantisation condition (7).

When matter in the fundamental representation is included the picture changes in detail, but is similar in structure. ${ }^{18}$ In particular different subgroups of $\Gamma(1)$ appear. To anticipate the notation we let $\Gamma_{0}(N) \subset \Gamma(1)$ denote the set of matrices with integral entries and unit determinant (12) such that $c=0 \bmod N$ and let $\Gamma^{0}(N) \subset \Gamma(1)$ denote those with $b=0 \bmod N$. These are both subgroups of $\Gamma(1)$ : $\Gamma_{0}(N)$ being generated by $\mathcal{T}$ and $\mathcal{S}^{-1} \mathcal{T}^{N} \mathcal{S}$ while $\Gamma^{0}(N)$ is generated by $\mathcal{T}^{N}$ and $\mathcal{S}^{-1} \mathcal{T S}$. 
Now consider $\mathcal{N}=2$ supersymmetric $\mathrm{SU}(2)$ Yang Mills theory in fourdimensional Minkowski space with $N_{f}$ flavours in the fundamental representation of $\mathrm{SU}(2)$. The low energy effective action for $0<N_{f}<4$ was derived in Ref. 18 . As the matter fields can have half-integral charges, it is convenient to re-scale the charge by a factor of two and define

$$
\tau^{\prime}=\frac{\theta}{\pi}+\frac{8 \pi i}{g^{2}}
$$

Thus

$$
\begin{gathered}
\gamma(\tau)=\tau+1 \Rightarrow \gamma\left(\tau^{\prime}\right)=2 \gamma(\tau)=\tau^{\prime}+2, \\
\gamma(\tau)=\frac{\tau}{1-2 \tau} \Rightarrow \gamma\left(\tau^{\prime}\right)=2 \gamma(\tau)=\frac{\tau^{\prime}}{1-\tau^{\prime}},
\end{gathered}
$$

and $\Gamma_{0}(2)$ acting on $\tau$ is equivalent to $\Gamma^{0}(2)$ acting on $\tau^{\prime}$.

The quantum modular symmetries of the scaling function acting on $\tau^{\prime}$ are

$$
\begin{aligned}
& N_{f}=0, \Gamma^{0}(2) \\
& N_{f}=1, \Gamma(1) \\
& N_{f}=2, \Gamma_{0}(2) \\
& N_{f}=3, \Gamma_{0}(4)
\end{aligned}
$$

and explicit forms of the corresponding modular functions are given in Ref. 19. For $N_{f}=1$ and $N_{f}=3$ the group is the same as the symmetry group acting on the effective action while for $N_{f}=0$ and $N_{f}=2$ it is larger, due to the $\mathbf{Z}_{2}$ action on the $u$-plane. Note the maximal case of $N_{f}=1$ where the full modular group is manifest at the quantum level, in this case the duality transformation $\tau^{\prime} \rightarrow-1 / \tau^{\prime}$ is symmetric and $\tau^{\prime}=i$ is a fixed point.

\section{Duality and the Quantum Hall Effect}

Modular symmetry manifests itself in the quantum Hall effect (QHE) in a manner remarkably similar to that of $\mathcal{N}=2$ supersymmetric Yang-Mills. But while it has not yet been established experimentally whether or not supersymmetry is relevant to the spectrum of elementary particles in Nature, the quantum Hall effect is rooted in experimental data and its supremely rich structure was not anticipated by theorists. The first suggestion that the modular group may be related to the QHE was in Ref. 20 but, as we shall see below, the wrong sub-group was identified in this earliest attempt.

The quantum Hall effect is a phenomenon associated with two-dimensional semiconductors in strong transverse magnetic fields at low temperatures, so that the thermal energy is much less than the cyclotron energy $\hbar \omega_{c}$, with $\omega_{c}$ the cyclotron frequency. It requires pure samples with high charge carrier mobility $\mu$ so that the dimensionless product $B \mu$ is close to unity. For reviews see e.g. Refs. 21-23. 
Basically passing a current $I$ through a rectangular two-dimensional slice of semiconducting material requires maintaining a voltage parallel to the current (the longitudinal voltage $V_{L}$ ). The presence of a magnetic field $B$ normal to the sample then generates a transverse voltage (the Hall voltage $V_{H}$ ). Two independent conductivities can therefore be defined: a longitudinal, or Ohmic, conductivity $\sigma_{L}$ and a transverse, or Hall, conductivity $\sigma_{H}$, along with the associated resistivities, $\rho_{L}$ and $\rho_{H}$. The classical Hall relation is

$$
B=-e \rho_{H} \Rightarrow \sigma_{H} B=J^{0}
$$

(with $J^{0}=e \mathbf{n}$ and $\mathbf{n}$ the density of charge carriers) and $\sigma_{H}$ is inversely proportional to $B$ at fixed $\mathbf{n}$. In the quantum Hall effect $\sigma_{H}$ is quantised as $1 / B$ is varied keeping $\mathbf{n}$ and $T$ fixed (or varying $\mathbf{n}$ keeping $B$ and $T$ fixed) and increases in a series of sharp steps between very flat plateaux. At the plateaux $\sigma_{L}$ vanishes and it is nonzero only for the transition region between plateaux. In two dimensions conductivity has dimensions of $e^{2} / h$ and, in the first experiments, ${ }^{24} \sigma_{H}=n \frac{e^{2}}{h}$ was an integral multiple of $e^{2} / h$ at the plateaux (the integer QHE) though in later experiments ${ }^{25}$ it was found that $\sigma_{H}$ could also be a rational multiple of $e^{2} / h, \sigma_{H}=\frac{p}{q} \frac{e^{2}}{h}$ where $q$ is almost always an odd integer (from now on we shall adopt units in which $e^{2} / h=1$ ). The different quantum Hall plateaux are interpreted as being different phases of a two-dimensional electron gas and transitions between the phases can be induced by varying the external magnetic field, keeping the charge carrier density constant.

Conductivity is actually a tensor

$$
J_{i}=\sigma_{i j} E^{j}
$$

with $\sigma_{x x}$ and $\sigma_{y y}$ the longitudinal conductivities in the $x$ and $y$ directions and $\sigma_{x y}=-\sigma_{y x}=\sigma_{H}$ the Hall conductivity associated with the magnetic field (for an elementary discussion of the Hall effect see Ref. 26). From now on we shall assume an isotropic medium with $\sigma_{x x}=\sigma_{y y}=\sigma_{L}$. Using complex coordinates $z=x+i y$ the conductivity tensor for an isotropic two-dimensional medium can be described by a single complex conductivity

$$
\sigma:=\sigma_{H}+i \sigma_{L}
$$

Note that Ohmic conductivities must be positive for stability reasons, so $\sigma$ is restricted to the upper-half complex plane. The resistivity tensor is the inverse of the conductivity matrix, in complex notation $\rho_{H}+i \rho_{L}=\rho=-1 / \sigma$.

The approach adopted here is that the response functions (i.e. the conductivities) in a low temperature two-dimensional system can be obtained from a $(2+1)$-dimensional field theory and to write down an effective action for $(2+1)$ dimensional electrodynamics in which all the microscopic physics associated with particles and/or holes has already been integrated out and incorporated into effective coupling constants. The classical Hall relation (29) can be derived from

$$
\mathcal{L}_{\text {eff }}\left[A_{0}\right]=-\sigma_{H} A_{0} B+A_{0} J^{0}
$$


the covariant version of which is

$$
\mathcal{L}_{\text {eff }}[A]=-\frac{\sigma_{H}}{2} \varepsilon^{\mu \nu \rho} A_{\mu} \partial_{\nu} A_{\rho}+A_{\mu} J^{\mu} .
$$

Ohmic conductivity can be incorporated by working in Fourier space $(\omega, \mathbf{p})$ and introducing a frequency dependent electric permittivity. In a conductor the low frequency electric permittivity diverges, in the long wavelength limit $\mathbf{p} \rightarrow 0$, as

$$
\varepsilon(\omega)=-i \frac{\sigma_{L}}{\omega}
$$

so, working in Fourier space, the effective dynamics of the electromagnetic field are governed by

$$
\begin{aligned}
\tilde{\mathcal{L}}_{\mathrm{cff}}[A] & =-\frac{\varepsilon}{4} F^{2}-\frac{\sigma_{H}}{4} \varepsilon^{\mu \nu \rho} A_{\mu} F_{\nu \rho}+A_{\mu} J^{\mu} \\
& \approx \frac{i \sigma_{L}}{4 \omega} F^{2}-\frac{\sigma_{H}}{4} \varepsilon^{\mu \nu \rho} A_{\mu} F_{\nu \rho}+A_{\mu} J^{\mu} .
\end{aligned}
$$

Note that the effective action is not real, an indication of the dissipative nature of Ohmic resistance, and non-local in time, again a feature of a conducting medium. Also we have used a relativistic notation and $F^{2}$ should really be split into $\mathrm{E} \cdot \mathrm{E}$ and $\mathbf{B} \cdot \mathbf{B}$ with different coefficients (response functions). In the long-wavelength, low-frequency limit of a conductor however, both response functions behave as $1 / \omega$, the ratio of the coefficients is a constant (the speed of light squared) and a relativistic notation can be used. ${ }^{\mathrm{f}}$ Chern Simons theories of the QHE have been considered by a number of authors. ${ }^{27-32}$ The inclusion of the $F^{2}$ term has been analysed from the general point of view of three-dimensional conformal field theory in Ref. 33.

The most relevant term in (35), in the renormalisation group sense, is the Chern Simons term and, at least naively assuming that there are no large anomalous dimensions arising from integrating out the microscopic degrees of freedom, the next most relevant term would be the usual Maxwell kinetic term. A version of the Lagrangian (35) was used in the analysis of Ref. 31 in which it was argued that the following transformations:

$$
\mathcal{T}: \sigma \rightarrow \sigma+1 \quad \text { and } \quad \mathcal{F}^{2}: \sigma \rightarrow \frac{\sigma}{1-2 \sigma}
$$

map between different quantum Hall phases of a spin polarised sample. ${ }^{\mathrm{g}}$ The $\mathcal{T}$ transformation is interpreted as being due to shifting Landau levels by one, e.g. by varying the magnetic field keeping $\mathbf{n}$ fixed. The $\mathcal{F}$ transformation, known as flux-attachment, was anticipated in Ref. 34 for $\sigma_{L}=0$ as a mapping between ground state wave functions. It is related to the composite fermion picture of the $\mathrm{QHE}^{22,35,36}$ where the effective mesoscopic degrees of freedom are fermionic particles which are composite objects consisting of more "fundamental" fermions bound

\footnotetext{
${ }^{\mathrm{f}}$ For finite $\omega$ and/or nonzero $\mathrm{p}$ this would not be the case as the response functions for $\mathrm{E}$ and $\mathrm{B}$ would be different functions in general.

g Neither complex conductivities nor relativistic notation was used in Ref. 31, but their results are most easily expressed in the notation used here.
} 
to an even number of magnetic flux-tubes of an effective U(1) gauge field known as the statistical gauge field. The operation $\mathcal{F}^{2}$ attaches two units of flux to each composite fermion.

The interpretation of the Hall plateaux in Ref. 31 is that one has charge carriers which are fermions (electrons or holes) interacting strongly with the external field. By attaching an odd number $2 k+1$ of statistical flux units to each fermion the resulting composite particles are bosons. By choosing $k$ appropriately, it can be arranged that the effect of the external magnetic field is canceled by the statistical gauge field and the composite bosons behave as free particles. Being bosons they can condense to form a superconducting phase with a mass gap and this explains the stability of the quantum Hall plateaux for the original fermions. Both the composite fermion and the composite boson picture are useful, but in either case the physical charge carriers are fermions.

The transformations (36) acting on the complex conductivity map between different quantum Hall phases, clearly they generate the group $\Gamma_{0}(2)$. As mentioned above the relevance of the modular group to the QHE was anticipated by Wilczek and Shapere, ${ }^{20}$ though these authors focused on a different subgroup of $\Gamma(1)$, one generated by $\mathcal{S}: \sigma \rightarrow-1 / \sigma$ and $\mathcal{T}^{2}: \sigma \rightarrow \sigma+2$ which the authors denoted by $\Gamma_{\theta}$, and the experimental data on the QHE do not bear this out, though we shall return to this group below. Lütken and Ross ${ }^{37}$ observed even before ${ }^{31}$ that the quantum Hall phase diagram in the complex conductivity plane bore a striking resemblance to the structure of moduli space in string theory, at least for toroidal geometry, and postulated that $\Gamma(1)$ was relevant to the quantum Hall effect. Later in Ref. 38 the subgroup $\Gamma_{0}(2)$ was identified as being one that preserves the parity of the denominator and therefore likely to be associated with the robustness of odd denominators.

Following the work of Ref. 31, see also Ref. 39, it was suggested in Ref. 40 that $\Gamma_{0}(2)$ should be the group relevant to two-dimensional systems involving spin polarised fermionic quasi-particles while Wilczek and Shapere's group $\Gamma_{\theta}$ should be relevant to two-dimensional systems involving bosonic quasi-particles. This led to a suite of predictions for two-dimensional superconductors in strong perpendicular magnetic fields described below. The analysis was generalised to the regime of nonlinear response in Ref. 41. Among the assumptions that go into the derivation of (36) from (35) are that the temperature is sufficiently low and that the sample is sufficiently pure, but unfortunately the analyses in Refs. 31,37, 40 and 41 are unable to quantify exactly what "sufficiently" means. Basically one must simply assume that (35) contains the most relevant terms for obtaining the long wavelength, low frequency response functions, but obviously this is not always true even at the lowest temperatures, for example it is believed that a Wigner crystal will form for filling fractions below about $1 / 7$ and (35), being rotationally invariant, cannot allow for this.

Of course $\Gamma_{0}(2)$ is not a symmetry of all of the physics, after all the conductivities differ on different plateaux, nevertheless it is a symmetry of some physical 
properties. The derivation of the $\Gamma_{0}(2)$ action in Ref. 31 required performing a Gaussian integral about a fixed background, different backgrounds give different initial conductivities, but in each case the dynamics contributing to the fluctuations are the same and the dynamics of the final system have the same form as that of the initial one. This motivates the suggestion ${ }^{42-44}$ that the scaling flow, which is governed by the fluctuations, should commute with $\Gamma_{0}(2)$, i.e. although $\Gamma_{0}(2)$ is not a symmetry of all the physics, it is a symmetry of the scaling flow. Physically the scaling flow of the QHE can be viewed as arising from changing the electron coherence length $l$, e.g. by varying the temperature $T$ with $l(T)$ a monotonic function of $T{ }^{45,46}$ Define a scaling function by

$$
\Sigma(\sigma, \bar{\sigma}):=l \frac{d \sigma}{d l} .
$$

Then, for any $\gamma \in \Gamma_{0}(2), \gamma(\sigma)=\frac{a \sigma+b}{c \sigma+d}$ with $a d-b c=1$, so

$$
\Sigma(\gamma(\sigma), \gamma(\bar{\sigma}))=\frac{1}{(c \sigma+d)^{2}} \Sigma(\sigma, \bar{\sigma}) .
$$

In general one expects $\sigma$ to depend on various parameters, such as the temperature $T$, the external field $B$, the charge carrier density $\mathbf{n}$ and the impurity density $\mathbf{n}_{i}$ among others. If $\mathbf{n}$ and $\mathbf{n}_{i}$ are fixed, then $\sigma(B, T)$ becomes a function of $B$ and $T$ only. Experiments show that fixed points between plateaux correspond to second order phase transitions between two quantum Hall phases and modular symmetry implies that scaling exponents should be the same at each fixed point, a phenomenon known as super-universality. While there is strong evidence for superuniversality ${ }^{47,48}$ there are some experiments which seem to violate $i t^{49}$ and this may be related to the suggestion that there exists a marginal operator at the critical point ${ }^{50}$ but an alternative explanation, that the scaling function might be a modular form, is proposed in Ref. 51.

A second striking consequence of $\Gamma_{0}(2)$ symmetry of the scaling flow is a selection rule for quantum Hall transitions. Given that the integer transition $\sigma: 2 \rightarrow 1$ is observed we conclude that $\gamma(2) \rightarrow \gamma(1)$ should also be possible. Let $\gamma(1)=\frac{q_{1}}{p_{1}}$ and $\gamma(2)=\frac{q_{2}}{p_{2}}$ where $q_{i}$ and $p_{i}$ are mutually prime $(i=1,2)$. Then, with $\gamma$ of the form (12), $\frac{q_{1}}{p_{1}}=\frac{a+b}{c+d}$ and $\frac{q_{2}}{p_{2}}=\frac{2 a+b}{2 c+d}$ so $q_{1}=a+b, p_{1}=c+d, q_{2}=2 a+b$ and $p_{2}=2 c+d$. Since $a d-b c=1$ we obtain the selection rule ${ }^{42}$

$$
q_{1} p_{2}-q_{2} p_{1}=1 .
$$

This selection rule is well borne out by the experimental data. In spin-split samples any two adjacent well-formed plateaux, with no unresolved sub-structure between them, obey this rule.

If we further assume that the only fixed points of the scaling flow are the fixed points of $\Gamma_{0}(2)$ then, with a few extra reasonable assumptions, the topology of the flow diagram is completely determined and it is exactly the same as the flow diagram in Fig. 1 for $\mathcal{N}=2$ SUSY. The whole upper-half plane can be covered by starting with the infinite strip of width 1 above the semi-circular arch spanning 0 and 1 in Fig. 1 (called the fundamental region of $\left.\Gamma_{0}(2)^{16}\right)$ and acting on it with 
elements of $\Gamma_{0}(2)$. Infrared fixed points have $\tau=q / p$ corresponding to fermionic charge carriers which are composite objects consisting of bosons with electric charge $q$ and $p$ units of statistical flux attached, with $p$ odd.

Assuming also, guided by experiment, that:

- rational numbers $q / p$ with odd $p$ are attractive, since they are experimentally so stable;

- the flow comes down vertically from the points at $\sigma=\sigma_{H}+i \infty$, i.e. that $\sigma_{H}$ does not flow at high temperatures (weak coupling ${ }^{51,52}$ )

then the topology forces the fixed points with $\Im \sigma>0$ to be saddle points and the only flow possible is compatible with the topology of Fig. 1. The precise form of the flow in Fig. 1 can be deformed, but the fixed points cannot be moved. An even stronger statement can be made with one further assumption. When a system is symmetric under particle-hole interchange one has symmetry under change of the sign of $\sigma_{H}$, which is $\sigma \rightarrow 1-\bar{\sigma}$ for the complex conductivity. This puts a reality condition on $\Sigma(\sigma, \bar{\sigma})$ which, combined with the mathematical properties of invariant functions of $\Gamma_{0}(2)$, can be used to show that the boundary of the fundamental domain, and its images under $\Gamma_{0}(2)$ must always be flow lines. ${ }^{53}$ Any deformation of Fig. 1 must therefore leave invariant the fixed points, the vertical lines above integers on the real axis and the semi-circles spanning rational numbers on the real line with odd denominators, (as well as the images of all these under $\Gamma_{0}(2)$ ) only the specific shape of the flow lines inside the fundamental domain, and their images under $\Gamma_{0}(2)$, can be deformed. In particular $\Gamma_{0}(2)$ symmetry provides a remarkably robust derivation of the well-established experimental semi-circle law. In many experiments the transition between two plateaux does follow a semi-circle in the upper-half complex conductivity plane to a very high degree of accuracy. ${ }^{54}$ The scaling hypothesis of Refs. 46 and 47 suggests that, at low temperatures, $\sigma$ becomes a function of a single scaling variable $\frac{B}{T}$ with a scaling exponent $\kappa$ and the flow is then forced onto one of these semi-circles. Any deviation from a semicircular transition is an experimental signal that the sample under investigation is not symmetric under particle-hole interchange. At a microscopic level the semicircle law has been derived in one specific model,,$^{55}$ but modular symmetry provides a very robust derivation, valid for any model which satisfies the above assumptions, and is therefore much more general than any specific model. The general topology of the flow in Fig. 1, at least for the integer QHE, was predicted by Khmel'nitskii, ${ }^{45}$ though the normalisation of the vertical axis was not determined in that analysis and there was no extension to the fractional case.

The specific flow for Fig. 1 was determined by supplementing the above assumptions with an additional constraint, that the scaling function $\Sigma(\sigma)$ should be a meromorphic function in the argument $\sigma{ }^{\mathrm{h}}$ This makes it a modular form of

h Strictly speaking it is meromorphic in the variable $q=e^{i \pi \sigma}$ rather than in $\sigma$ itself. 


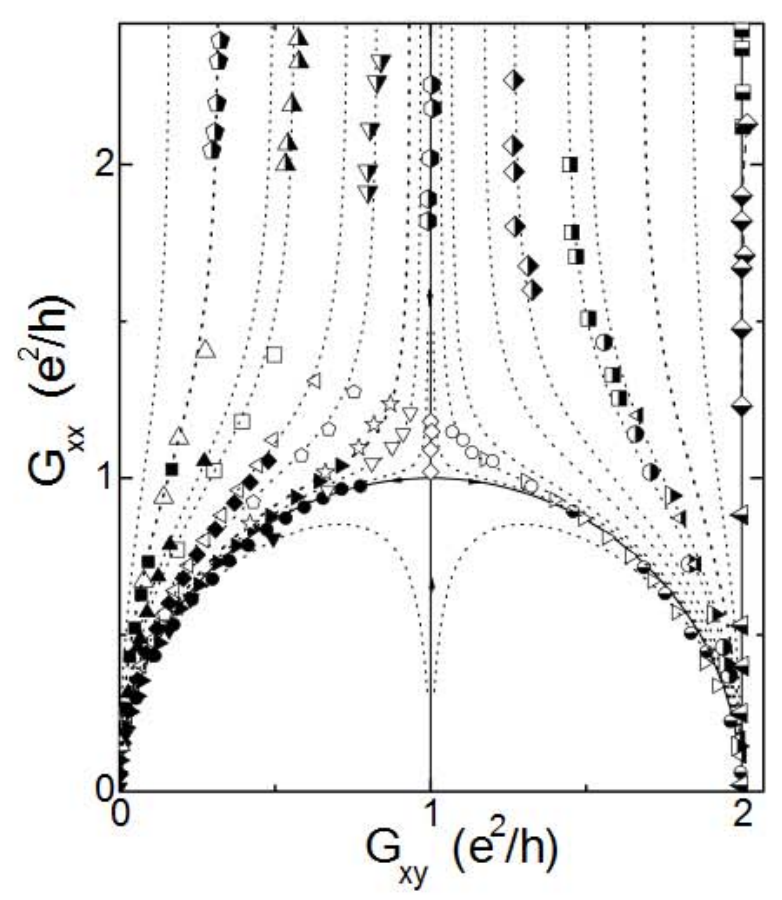

Fig. 2.

weight -2 and the analytic form that approaches the stable fixed points on the real axis most rapidly is exactly the same as that of $\mathcal{N}=2$ SUSY Yang Mills in the previous section, up to an undetermined constant, namely

$$
\Sigma(\sigma)=\frac{2}{\pi i} \frac{1}{\left(v_{3}^{4}(\sigma)+v_{4}^{4}(\sigma)\right)},
$$

and this gives the flow plotted in Fig. 1. To date there is no physical reason for assuming that $\Sigma$ should be independent of $\bar{\sigma}$, it is only motivated by mathematical analogy with SUSY Yang Mills, but it does have the advantage of giving an explicit form for the scaling function that can be visualised. Any deformation away from this by including a non-meromorphic component is constrained by the considerations above and cannot change the topology. In fact the flow obtained using the meromorphic ansatz does give remarkably good agreement with experiment and the comparison is plotted in Figs. 2 and 3, taken from Refs. 56 and 57. Further experimental evidence supporting modular symmetry in the QHE is given in Ref. 58 .

In summary, modular symmetry $\Gamma_{0}(2)$ applied to the quantum Hall effect for spin-split samples leads to the following predictions:

- universal critical points are predicted at $\sigma_{*}=\frac{1}{2}(1+i)$ and its images under $\Gamma_{0}(2)$. Critical exponents must be the same for all fixed points which are related by $\Gamma_{0}(2)$. 

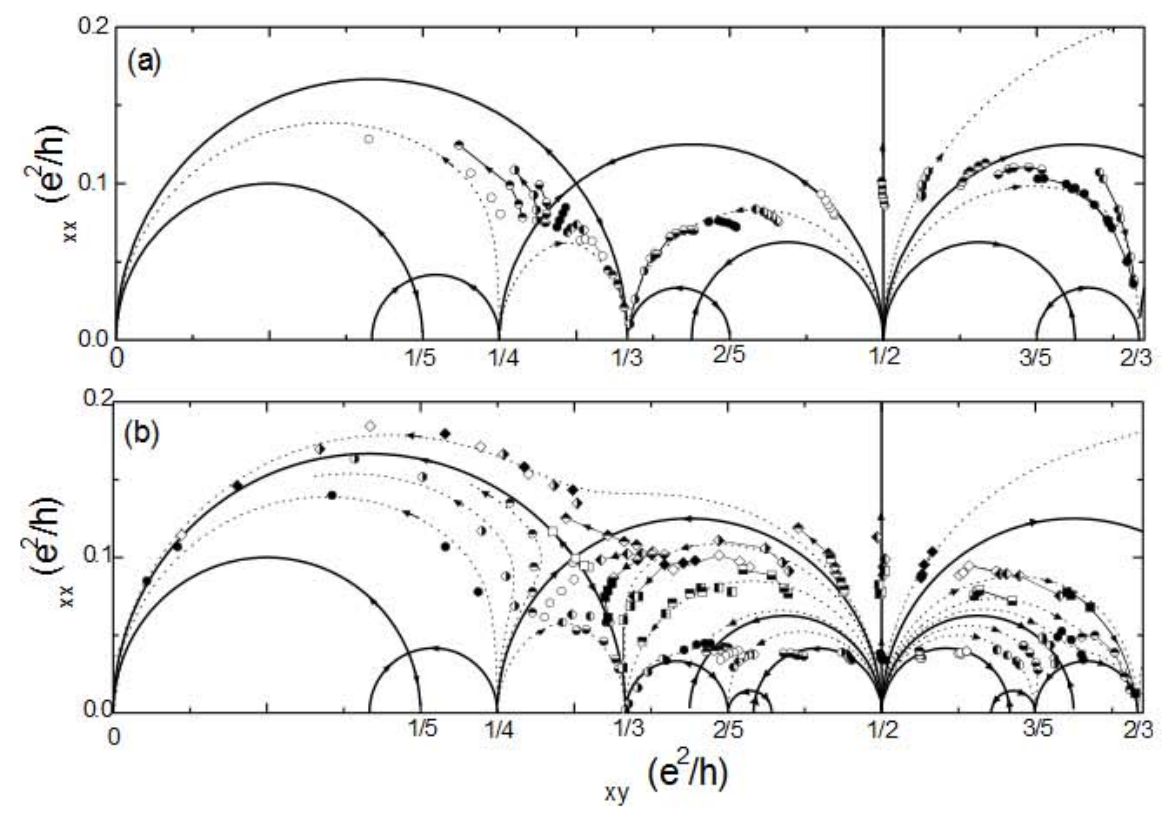

Fig. 3.

- Exact flow lines in the $\sigma$-plane can be derived from $\Gamma_{0}(2)$ invariance plus invariance under particle-hole symmetry: $\sigma \rightarrow 1-\bar{\sigma}_{*}$. In particular the semi-circle law is a consequence of $\Gamma_{0}(2)$ plus particle-hole symmetry.

- Flow in the infrared is towards the real axis, terminating on the real axis at attractive fixed points at odd-denominator fractions. Even-denominator fractions form repulsive fixed points of the flow.

- The selection rule $\left|p_{1} q_{2}-p_{2} q_{1}\right|=1$ for allowed transition between $\sigma=q_{1} / p_{1}$ and $\sigma=q_{2} / p_{2}$.

For QHE samples that are not fully spin polarised one expects that the Landau levels can come in adjacent pairs and $\mathcal{T}$ in (36) should be replaced by $\mathcal{T}^{2}$, giving the generators of $\Gamma(2)$ rather than $\Gamma_{0}(2)$, and this modifies the experimental consequences. $\Gamma(2)$ actually has no fixed points above the real axis, so one cannot make any predictions about the position of the second-order phase transition between two plateaux, but if there is particle-hole symmetry one still has the semi-circle law and the fixed point must lie somewhere on the semi-circle ${ }^{59}$ An experimental analysis of the relation between modular symmetry and Zeeman splitting is given in Ref. 60 .

The group $\Gamma(2)$ was also analysed, from the point of view of its action on ground state wave-functions rather than on complex conductivities, in Ref. 61 and on complex conductivities in Ref. 62. Jain's map was described in terms of duality and mirror symmetry in Ref. 63. 
The group $\Gamma_{0}(2)$ is the subgroup of the full modular group that is relevant for systems with fermionic charge carriers in a strong perpendicular magnetic field with the spins well split. If the effective charge carriers are bosonic, e.g. two-dimensional superconductors, one can obtain predictions from the fermionic case by using the flux attachment transformation to add an odd number of vortices to the quasiparticles. For the case of a single unit of flux, this equivalent to conjugating $\Gamma_{0}(2)$ by $\mathcal{F}=\mathcal{S}^{-1} \mathcal{T} \mathcal{S}$. The resulting group is generated by $\mathcal{S}$ and $\mathcal{T}^{2}$ and is the group $\Gamma_{\theta}$ of Refs. 20, 40 and 41 (a derivation of the combination of the $\mathcal{S}$ transformation with particle-hole symmetry, for a two-dimensional superconductor, was given in Ref. 64). This group consists of elements $\gamma$ of the form (12) with either $a, d$ both odd and $b, c$ both even, or vice versa. In this case the predictions are different:

- universal critical points are predicted for the flow at the fixed points for transitions between stable phases, $\sigma_{*}=i$ and its images under $\Gamma_{\theta}{ }^{\mathrm{i}}$

- The critical exponents at all fixed points related by $\Gamma_{\theta}$ must all be the same.

- Exact flow lines in the $\sigma$-plane are immediate consequences of $\Gamma_{\theta}$ invariance and particle-hole symmetry. ${ }^{40}$ The results are again semi-circles or vertical lines in the $\sigma$-plane, implying a semi-circle law for these bosonic systems.

- For nonzero magnetic fields the flow as the temperature is reduced is towards the real axis, terminating on the attractive fixed points $\sigma=p / q$ with $p q$ even (as opposed to having $q$ odd, as was the case for fermions). Fractions with odd $p q$ are repulsive fixed points.

- There is a selection rule that allowed fractions $p_{2} / q_{2}$ can be obtained from $p_{1} / q_{1}$ only if $\left|p_{1} q_{2}-p_{2} q_{1}\right|=1$.

The resulting flow diagram for bosonic systems is given in Ref. 40 , it has a fixed point at $\sigma_{*}=i$, as predicted by Fisher. ${ }^{65}$ To date no two-dimensional superconductors have been manufactured with a high enough mobility $\mu$ that $\mu B$ is close to unity for sustainable magnetic fields, but it is predicted in Ref. 40 that a hierarchy with the above properties will be observed if such samples are ever manufactured.

\section{Conclusions}

Modular symmetry is a generalisation of the Dirac quantisation condition for charge in QED. Its mathematical foundation is strongest in supersymmetric systems, such as supersymmetric Yang-Mills and string theory, but a more realistic system, with a wealth of experimental data to compare with, is the quantum Hall effect. The Dirac-Schwinger-Zwanziger quantisation condition manifests itself in quantum Hall systems as a selection rule for transitions between allowed quantum Hall plateaux.

An important feature that these systems have in common is the existence of topologically non-trivial field configurations, monopoles in SUSY Yang Mills and

iThis statement is for bosonic charge carriers with the same electric charge as an electron - it becomes $\sigma_{*}=i \tilde{q}^{2}$ and its images if the bosonic charge carriers have charge $\tilde{q}$. In particular, the case $\tilde{q}= \pm 2$ applies if the bosons are Cooper pairs, such as those considered in Ref. 65. 
vortices in the QHE, which can bind to the charge carriers to form pseudo-particles carrying magnetic charge: dyons in SUSY Yang Mills and in the QHE fermions which can be viewed as bosons with an odd number of flux units attached. The rational nature of the attractive fixed points on the real axis $q / p$ is then related to electric charge $q$ and magnetic charge $p$.

The earliest appearance of modular symmetry in the condensed matter literature was in the work of Cardy and Rabinovicci, ${ }^{66}$ where a coupled clock model was analysed, interestingly with a view to gaining insight into quantum chromodynamics. Phase diagrams for clock models were related to the QHE in Ref. 67. Another model in which the modular group was found is the dissipative Hofstader model. ${ }^{68}$ There are certain features which all these models have in common. They all have only two relevant couplings: one, $y$, associated with the dynamical kinetic term, which must be positive for stability reasons; and one, $x$, associated with a topological term. These are combined into the complex parameter, $\tau=x+i y$, on the upper-half complex plane on which the modular group, or a subgroup thereof, acts. It seems that modular symmetry is not just an accident of one type of system, or even a family of systems such as supersymmetric field theories, but in fact is a more general phenomenon and it may yet prove even more powerful in understanding the physics of strongly interacting systems.

\section{References}

1. P. A. M. Dirac, Proc. Roy. Soc. A 133, 60 (1931).

2. J. Schwinger, Phys. Rev. 144, 1987 (1966); D. Zwanziger, Phys. Rev. D 3, 880 (1971).

3. E. Witten, Phys. Lett. B 86, 283 (1979).

4. E. Witten, Selecta. Math. (NS) 1, 383 (1995); E Verlinde, Nucl. Phys. B 455, 211 (1995).

5. N. Seiberg and E. Witten, Nucl. Phys. B 426, 19 (1994) [Erratum, ibid. 430, 169 (1994)].

6. L. Alvarez-Gaume and S. F. Hassan, Fortsch. Phys. 45, 159 (1997).

7. M. E. Peskin, hep-th/ 9702094 .

8. N. Dorey, V. V. Khoze and M. P. Mattis, Phys. Rev. D 54, 2921 (1996).

9. J. A. Minahan and D. Nemeschansky, Nucl. Phys. B 468, 72 (1996).

10. A. Ritz, Phys. Lett. B 434, 54 (1998).

11. B. P. Dolan, Phys. Lett. B 418, 107 (1998).

12. G. Carlino, K. Konishi, N. Maggiore and N. Magnoli, Phys. Lett. B 455, 171 (1999); K. Konishi, Int. J. Mod. Phys. A 16, 1861 (2001).

13. E. T. Whittaker and G. N. Watson, A Course of Modern A nalysis (Cambridge Univ. Press, 1940).

14. E. D'Hoker, I. M. Krichever and D. H. Phong, Nucl. Phys. B 489, 179 (1997); ibid. 494, 89 (1997).

15. M. Matone, Phys. Lett. B 357, 342 (1995).

16. R. A. Rankin, Modular Forms and Functions (Cambridge Univ. Press, 1977).

17. B. P. Dolan, hep-th/ 0505138.

18. N. Seiberg and E. Witten, Nucl. Phys. B 431, 484 (1994).

19. B. P. Dolan, Nucl. Phys. B 737, 153 (2006).

20. A. Shapere and F. Wilczek, Nucl. Phys. B 320, 669 (1989). 
21. The Quantum Hall E ect, eds. R. E. Prange and S. M. Girvin (Springer, 1987).

22. Composite Fermions, ed. O. Heinonen (World Scienti c, 1998).

23. M. Stone, Quantum Hall E ect (World Scienti C, 1992).

24. K. von Klitzing, G. Dorda and M. Pepper, Phys. Rev. Lett. 45, 494 (1980).

25. D. C. Tsui, H. L. Stormer and A. C. Gossard, Phys. Rev. Lett. 48, 1559 (1982).

26. L. Landau and E. Lifschitz, A Course in Theoretical Physics, Vol. 8, 2nd edn. (Pergamon Press, 1984).

27. S. M. Girvin, in Ref. 21.

28. S. C. Zhang, T. H. Hansson and S. Kivelson, Phys. Rev. Lett. 62,82 (1989).

29. J. Frohlich and A. Ze, Nuc. Phys. B 364, 517 (1991).

30. A. P. Balachandran and A. M. Srivastava, hep-th/ 9111006.

31. S. Kivelson, D.-H. Le and S.-C. Zhang, Phys. Rev. B 46, 2223 (1992).

32. A. Balatsky and E. Fradkin, Phys. Rev. B 43, 10622 (1991); E. Fradkin, Nucl. Phys. B 474 [F S], 543 (1996).

33. E. Witten, hep-th/ 0307041; R. G. Leigh and A. C. Petkou, JHEP 0312, 020 (2003): R. Zucchini, Adv. Theor. Math. Phys. 8, 895 (2005); H.-U. Ye, Phys. Lett. B 598, 139 (2004).

34. J. K Jain, S. A. Kivelson and N. Trivedi, Phys. Rev. Lett. 64, 1927 (1990); J. K. Jain and V. J. Goldman, Phys. Rev. B 45, 1255 (1992).

35. J. K. Jain, Phys. Rev. Lett. 63, 199 (1989); Phys. Rev. B 41, 7653 (1990).

36. A. Lopez and E. Fradkin, Phys. Rev. B 44, 5246 (1991).

37. C. A. Lutken and G. Ross, Phys. Rev. B 45, 11837 (1992); C. A. Lutken and G. Ross, Phys. Rev. B 48, 2500 (1993).

38. C. A. Lutken, J. Phys. A 26, L811 (1993); Nucl. Phys. B 396, 670 (1993).

39. L. P. Pryadko, Phys. Rev. B 56, 6810 (1997).

40. C. Burgess and B. P. Dolan, Phys. Rev. B 63, 155309 (2001).

41. C. Burgess and B. P. Dolan, Phys. Rev. B 65, 155323 (2002).

42. B. P. Dolan, J. Phys. A 32, L243 (1999).

43. N. Taniguchi, cond-mat/ 9810334 .

44. C. Burgess and A. Lutken, Nucl. Phys. B 500, 367 (1997); Phys. Lett. B 451, 365 (1999).

45. D. E. Khmel'nitskii, Pis'ma Zh. Eksp. Teor. Fiz 38, 454 (1983) [JETP Lett. 38, 552 (1983)].

46. A. M. M. Pruisken, Phys. Rev. Lett. 61, 1297 (1988).

47. H. P. Wei, D. C. Tsui, M. A. Paalanen and A. M. M. Pruisken, Phys. Rev. Lett. 61, 1294 (1988).

48. L. Engel, H. P. Wei, D. C. T sui and M. Shayegan, Surf. Sci. 229, 13 (1990).

49. D. Shahar, M. Hilke, C. C. Li, D. C. Tsui, S. L. Sondhi and M. Razeghi, Solid State Commun. 107, 19 (1998).

50. M. R. Zirnbauer, hep-th/ 9905054 .

51. B. P. Dolan, Nucl. Phys. B 554, 487 (1999).

52. A. M. M. Pruisken, in Ref. 21.

53. C. Burgess, B. P. Dolan and Rim Dib, Phys. Rev. B 62, 15359 (2000).

54. M. Hilke et al., Eur. Phys. Lett. 46, 775 (1999).

55. A. M. Dykhne and I. M. Ruzin, Phys. Rev. B 50, 2369 (1994); I. Ruzin and S. Feng, Phys. Rev. Lett. 74, 154 (1995).

56. S. S. Murzin, M. Weiss, A. G. M. Jansen and K. Eberl, Phys. Rev. B 66, 233314 (2002).

57. S. S. Murzin, S. I. Dorozhkin, D. K. Maude and A. G. M. Jansen, Phys. Rev. B 72, 195317 (2005) 
58. C. F. Huang, Y. H. Chang, H. H. Cheng, C.-T. Liang and G. J. Hwang, Physica E 22, 232 (2004).

59. B. P. Dolan, Phys. Rev. B $62,10278(2000)$.

60. D. R. Hang et al., Micrœlectron. J. 36, 469 (2005).

61. M. A. I. Flohr, hep-th/ 9412053.

62. Y. Georgelin, T. Masson and J.-C. Wallet, J. Phys. A 33, 39 (1990); Y. Georgelin and J.-C. Wallet, Phys. Lett. A 224, 303 (1997); Y. Georgelin, T. Masson and J.-C. Wallet, J. Phys. A 30, 5065 (1997).

63. I. Kogan and R. Szabo, Phys. Rev. B 58, 7893 (1998).

64. S.-J. Rey and A. Ze, Nucl. Phys. B 352, 897 (1991).

65. M. P. A. Fisher, Phys. Rev. Lett. 65, 923 (1990).

66. J. L. Cardy and E. Rabinovici, Nucl. Phys. B 205, 1 (1982); J. L. Cardy, ibid. 205 , 17 (1982).

67. M. A sorey, J. G. Esteve and J. Salas, Phys. Rev. B 48, 3626 (1993).

68. C. G. Callan and D. Fred, Nucl. Phys. B 374,543 (1992); C. Callan, A. Felce and D. Freed, ibid. 392, 551 (1993). 